\title{
BETA-BLOCKER TREATMENT GUIDED BY HEAD-UP TILT TEST IN NEURALLY MEDIATED SYNCOPE
}

\author{
GIUSEPPE BORIANI, MAURO BIFFI, GABRIELE BRONZETTI, PAOLO SABBATANI, \\ ANGELO BRANZI, AND BRUNO MAGNANI
}

Institute of Cardiology, University of Bologna, Bologna, Italy

\begin{abstract}
This study was an open-label, uncontrolled, dose-escalation trial of beta-blockers in patients with a history of syncope without warning or syncope resulting in trauma (malignant vasovagal syncope) who had positive head-up tilt test (HUT) responses, with or without isoproterenol infusion. Thirty patients (mean age, $37 \pm 21$ years) with recurrent syncopal and near-syncopal episodes of unexplained origin in the previous year $(6 \pm 14$ syncopal episodes and $17 \pm 3$ nearsyncopes) underwent HUT for diagnostic purposes and for guiding prophylactic treatment. After patients were given a 10-minute rest in a recumbent position, we performed an HUT at $70^{\circ}$ for 25 minutes; if indicated, isoproterenol testing was performed at incremental dosages (five steps at 10 -minute intervals at $80^{\circ}$ ). All patients experienced syncope during HUT, $15(50 \%)$ at baseline HUT and 15 (50\%) during isoproterenol infusion ( 1 to $3 \mathrm{\mu g} / \mathrm{min}$; mean, $1.6 \mathrm{\mu g} /$ min). Sixteen syncopes were of vasodepressor type, 10 were mixed, and 4 were of cardioinhibitory type. After baseline HUT, betablocking drugs were prescribed to all patients as follows: 1 patient was given propranolol (160 mg daily), and 29 patients were given metoprolol ( $246 \pm 49 \mathrm{mg}$ daily), with a dose titration period of 14 days. HUT was repeated after 3 weeks, and 24 patients $(80 \%)$ had negative results (no syncope or anomalous responses). After further dosage adjustment of beta-blockers in nonresponders, a negative HUT was obtained in 28 patients (93\%). Overall mean metoprolol daily dose was $262 \pm 60 \mathrm{mg}$ ( 29 patients), and propranolol was administered at $160 \mathrm{mg}$ daily in 1 patient. Thirteen patients (43\%) reported side effects, none of which required drug withdrawal. At an average follow-up of $16 \pm 1$ months, none of the patients experienced syncope, a statistically significant reduction. Moreover, a statistically significant reduction in the number of near-syncopal episodes was observed in comparison to the previous year. None of the patients discontinued treatment because of long-term side effects. Beta-blockers were well tolerated and achieved a high rate of efficacy, even in cardioinhibitory syncopes. In conclusion, in selected patients with malignant vasovagal syncope, treatment with metoprolol or propranolol at relatively high doses is feasible and, if
\end{abstract}

Address correspondence to: Dr. Giuseppe Boriani, Institute of Cardiology, University of Bologna, Policlinico S. Orsola via Massarenti $n^{\circ}$ 9, 40138 Bologna, Italy.

Received for publication on August 4, 1997. Printed in the U.S.A.

Reproduction in whole or part is not permitted. 
guided by HUT results, is associated with a favorable outcome in terms of freedom from syncopal recurrences. Appropriate titration to achieve the full beta-blocking effect appears to be advisable. Key words: beta-blockers, head-up tilt test, neurally mediated syncope.

\section{INTRODUCTION}

The head-up tilt test (HUT) is widely used to diagnose neurally mediated syncope and has been proposed as a means for evaluating drug therapy for such syncopal episodes, ${ }^{1}$ although a low recurrence rate after placebo treatment has been reported by some authors. ${ }^{2-7}$

Among patients with neurally mediated syncope, some experience syncope without warning or prodromes. In these patients syncope may be associated with injury or trauma and is called malignant vasovagal syncope. ${ }^{8,9} \mathrm{We}$ believe that prophylactic treatment is strongly indicated in this subgroup of patients. Various pharmacologic treatments and even dualchamber pacing have been used for this purpose, and HUT has been proposed as a guide to long-term prophylaxis. ${ }^{7}$

The aim of this study was to assess the efficacy and tolerability of treatment with beta-blockers, guided by HUT, in patients with syncope associated with trauma or injuries or without prodromes. Specifically, our aim was to titrate the dosage of beta-blockers to levels higher than those used by previous researchers. ${ }^{7}$

\section{PATIENTS AND METHODS}

\section{Patient Selection}

Patients referred to our laboratory for HUT as part of the evaluation for unexplained syncope were eligible for this study. Baseline cardiac evaluation included a chest roentgenogram, cross-sectional echocardiogram, and, if clinically requested, Holter monitoring and an exercise test. A clinical history was obtained from each patient, and the circumstances surrounding previous syncopal episodes were reviewed. Presyncope, or near-syncope, was defined as an episode of near-loss of consciousness. Patients with a positive HUT were included in this study only if clinical syncopal episodes were associated with injuries or trauma or occurred without prodromes. Verbal informed consent was obtained from all adult patients and from parents of patients under 18 years of age.

\section{Methods}

Patients were studied between $9 \mathrm{AM}$ and noon after an overnight fast and during a washout from any cardioactive drug for at least five half- 
lives. Patients were placed supine on the tilt table, and an intravenous catheter was inserted in a peripheral vein. Blood pressure was measured by means of an arterial cuff at baseline and then continuously monitored noninvasively (Finapress Ohmeda, Englewood, Colorado). After a resting period of 10 minutes, patients were tilted upright to $70^{\circ}$ during continuous electrocardiographic monitoring and maintained in that position for 25 minutes unless syncope occurred. If patients completed this stage without a positive response, they were returned to the supine position and underwent HUT while receiving isoproterenol. Isoproterenol testing included five steps, each preceded by 10 minutes of recumbency, during which isoproterenol was infused at increasing rates (from 1 to $5 \mu \mathrm{g} / \mathrm{min}$ ), and patients were tilted to $80^{\circ}$ for 10 minutes. A positive response to HUT was defined as sudden loss of consciousness or the development of presyncope in association with an abrupt decrease in systolic blood pressure to $\leqslant 60$ $\mathrm{mm} \mathrm{Hg}$, whereas completion of the baseline protocol and isoproterenol testing without these responses constituted a negative response to HUT. The hemodynamic pattern of the response (cardioinhibitory, vasodepressive, or mixed) was defined according to VASIS classification. ${ }^{8}$

\section{Treatment with Beta-Blockers}

Patients with a positive HUT who fulfilled the previously mentioned requirements (clinical syncope without prodromes or associated with trauma), consistent with the definition of malignant vasovagal syncope, began treatment with a beta-blocker (metoprolol or propranolol). Prophylactic treatment was strongly indicated in these highly selected patients, and we considered it unethical to either give a placebo or avoid active treatment. Initial daily dosages were $50 \mathrm{mg}$ for metoprolol and $80 \mathrm{mg}$ for propranolol. The dosages were titrated during a 14-day period to reach at least $100 \mathrm{mg}$ twice a day (BID) for metoprolol and $160 \mathrm{mg}$ for propranolol, or to the maximum tolerated dosage. After 3 weeks, patients underwent another HUT, including isoproterenol. If this HUT result was positive or patients experienced recurrence of syncope or presyncope, a further increase in the dose of beta-blockers was considered.

Data concerning the occurrence of adverse events were collected by assessing patients' complaints, and the possible necessity of drug withdrawal was evaluated at every visit.

\section{Long-Term Follow-up}

According to the results of HUT performed under oral treatment with beta-blockers, all patients were eligible for long-term prophylaxis. They 
were followed up in an outpatient clinic every 3 months and were requested to report the occurrence of any episode of syncope or near-syncope. Patients' reports of adverse events were recorded during the follow-up period.

\section{Statistical Analysis}

Data are expressed as mean \pm SD. Student's paired $t$ test was used to compare variables before and after treatment. A $P$ value $<0.05$ was considered statistically significant.

\section{RESULTS}

Thirty patients 12 to 82 years of age (mean age, $37 \pm 21$ years) with syncope without prodromes or associated with trauma or injuries were enrolled for the baseline HUT. Twenty-eight (93\%) of the 30 patients had no evidence of structural heart disease, and 2 patients (7\%) had mitral valve prolapse with trivial mitral regurgitation. The number of syncopal and presyncopal episodes experienced in the previous year is shown in Table I.

\section{Baseline Head-up Tilt Test Response}

At baseline, all 30 patients experienced syncope (during HUT in 15 [50\%] of the patients and during isoproterenol testing [mean isoproterenol infusion rate $1.6 \mu \mathrm{g} / \mathrm{min}$; range, 1 to $3 \mu \mathrm{g} / \mathrm{min}$ ] in 15 [50\%] of the patients). The pattern of response during HUT was vasodepressor type in 16 patients $(53 \%)$, mixed type in 10 patients $(33 \%)$, and cardioinhibitory type in 4 patients (13\%) (mean asystole, $13 \pm 5$ seconds; range, 7 to 19 seconds).

Table I. Data on long-term follow-up ( $16 \pm 4$ months) during treatment with beta-blockers $(\mathrm{N}=28)$.

\begin{tabular}{lcc}
\hline \multicolumn{1}{c}{ Clinical Event } & Previous Year & Follow-up \\
\hline Syncopal episodes/patient (mean \pm SD) & $6 \pm 14$ & $0^{\star}$ \\
Syncopal episodes in all patients (total) & 214 & $0^{\star}$ \\
Near syncops/paticnt (mean $\perp$ SD) & $17 \pm 3$ & $0.3 \pm 0.05^{*}$ \\
Near syncopes in all patients (total) & 327 & $11^{*}$ \\
\hline \hline
\end{tabular}

${ }^{*} P<0.001$ versus previous year. 


\section{Treatment with Beta-Blockers and Control Head-up Tilt Test Response}

All patients were treated with beta-blockers; $29(97 \%)$ of the 30 patients received metoprolol and 1 patient (3\%) received propranolol. $\mathrm{Pa}-$ tients in the metoprolol group began the treatment with $25 \mathrm{mg} \mathrm{BID}$, with progressive titration based on clinical evidence of full-degree betablockade. At the end of the titration period, the daily dosage for metoprolol ranged from 75 to $200 \mathrm{mg}$ BID (mean daily dosage, $246 \pm 69 \mathrm{mg}$ ). Specifically, 1 patient received $100 \mathrm{mg}$; 3 patients, $150 \mathrm{mg} ; 10$ patients, $200 \mathrm{mg}$; 14 patients, $300 \mathrm{mg}$; and 1 patient, $400 \mathrm{mg}$. One patient received propranolol $160 \mathrm{mg}$ daily.

At a control HUT performed after 3 weeks, 24 patients $(80 \%)$ had negative results without syncope or anomalous responses. In 4 patients (13\%) with a persistently positive HUT during beta-blockade coupled with spontaneous recurrences of syncope, a further increase in beta-blocking dosage was attempted (Table II), and HUT was repeated with the new dosage, with satisfactory results (negative HUT). Overall, a negative HUT was obtained in 28 patients (93\%). Nonresponders to beta-blocker therapy included 1 patient with a persistent mixed response (after metoprolol, HUT was positive only during isoproterenol testing) and 1 patient with a persistent vasodepressive response during isoproterenol testing, similar to the baseline HUT. After dosage adjustment, mean daily dosage for metoprolol was $262 \pm 60 \mathrm{mg}$ (29 patients).

\section{Long-Term Follow-up}

The 28 patients who responded to HUT-guided therapy with betablockers (after titration in 4 patients) were followed up for $16 \pm 4$ months.

Table II. Effects of further increase in beta-blocker dosage on head-up tilt test (HUT) response in four patients with persistently positive HUT results.

\begin{tabular}{|c|c|c|c|c|}
\hline $\begin{array}{c}\text { Patient Sex, } \\
\text { Age (y) }\end{array}$ & Clinical Picture & Drug & $\begin{array}{c}\text { Dosage at HUT } \\
\text { Posilive } \\
\text { (mg/d) }\end{array}$ & $\begin{array}{c}\text { Dosage at HUT } \\
\text { Negative } \\
\text { (mg/d) }\end{array}$ \\
\hline$F, 27$ & $\begin{array}{l}\text { VD syncope with } \\
\text { mandibular fracture }\end{array}$ & Propranolol & 160 & 320 \\
\hline$M, 55$ & $\begin{array}{l}\text { VD syncope with } \\
\text { facial trauma }\end{array}$ & Metoprolol & 200 & 300 \\
\hline $\mathrm{F}, 22$ & $\begin{array}{l}\text { Mixed syncope } \\
\text { (1 syncope/month) }\end{array}$ & Metoprolol & 150 & 300 \\
\hline$M, 16$ & $\begin{array}{l}\text { Mixed syncope } \\
\text { with trauma }\end{array}$ & Metoprolol & 100 & 250 \\
\hline
\end{tabular}

VD = vasodepressor 
As shown in Table I, a significant reduction in the number of syncopal or near-syncopal episodes was observed in comparison to the previous year $(P<0.001)$. At follow-up (3 months after HUT), heart rate was reduced from $74 \pm 9$ beats $/ \mathrm{min}$ (baseline) to $50 \pm 6$ beats $/ \mathrm{min}(P<0.005)$, whereas mean cuff blood pressure was statistically unchanged (from $96 \pm 8 \mathrm{~mm} \mathrm{Hg}$ to $94 \pm 7 \mathrm{~mm} \mathrm{Hg}$ ).

\section{Adverse Events}

Treatment-related side effects occurring early (within 3 weeks) or late (during long-term follow-up) were evaluated. Among the former, 13 patients $(43 \%)$ experienced side effects: 7 patients experienced fatigue on exertion, which improved with physical training; 3 patients had palpitations, which disappeared by increasing the beta-blocker dosage; 2 patients experienced an increase in dreams, which was judged as tolerable; and 1 patient had cold extremities. During follow-up, 1 patient had persistent mild fatigue and 1 patient continued to experience dreams. No drug withdrawal was required because of early or late side effects.

\section{DISCUSSION}

Vasovagal syncope may occur with different characteristics ranging from common faints preceded by prodromes to syncope with minimal or no warning, often associated with trauma or injuries. The term malignant vasovagal syncope has been used for the latter form of syncope, ${ }^{9}$ and, because of the potential risks, ${ }^{10}$ prophylactic treatment is required in these patients. HUT is the gold standard for establishing the diagnosis of neurally mediated syncope; however, issues remain about methodology, reproducibility, and the role of serial HUT in the selection of patients for prophylactic treatment. ${ }^{2}$ According to previous studies, ${ }^{11-14}$ our protocol (baseline HUT followed by short increases in the rate of isoproterenol infusion) produced results that were reproducible and reliable, and this is the basis for assessing the efficacy of a prophylactic treatment.

A low syncopal recurrence rate has been reported in the follow-up of patients with vasovagal syncope not treated with active drugs. ${ }^{2-6}$ In this study, however, we assessed a selected subgroup of patients in whom syncope was associated with trauma and injuries or occurred without warning or prodromes. Prophylactic treatment was strongly indicated in this subgroup, and we adopted beta-blocker therapy guided by HUT results. Previous studies have proposed various options, including dual-chamber pacing, ${ }^{15,16}$ for preventing neurally mediated syncope.

The present study showed how, in selected patients, suppression of HUT-induced syncope is related to a favorable outcome during follow-up. 
Moreover, HUT-guided treatment may be helpful because careful titration of beta-blockers is advisable and because increasing the dosages of betablockers after a positive HUT may lead to a better drug response and a more favorable outcome. In our experience, relatively high doses of betablocking drugs are required to achieve negative responses with HUT, and this finding is consistent with the proposed pathophysiologic hypothesis for vasovagal syncope, ${ }^{17,18}$ because inhibition of increased myocardial inotropy is required in these patients.

The mean dosage of beta-blockers (primarily metoprolol) used in this study is higher than the dosage of metoprolol or atenolol used in other studies, where atenolol was prescribed at $50 \mathrm{mg}$ once daily ${ }^{5,6,19}$ or metoprolol at 25 to $50 \mathrm{mg} \mathrm{BID} .{ }^{20}$ In a study by Mahanonda et al, ${ }^{21}$ atenolol showed a substantial decrease in the recurrence of symptoms in a randomized, controlled trial of 42 patients who received 50 to $100 \mathrm{mg}$ of the drug daily. In that study, ${ }^{21} 62 \%$ of patients receiving atenolol had a negative HUT compared with $5 \%$ receiving placebo, and atenolol efficacy results were even higher ( $38 \%$ of patients), as reported by Fitzpatrick et al. ${ }^{5}$ The likelihood of dose-related differences in HUT response in patients receiving atenolol was not investigated by Mahanonda et $\mathrm{al}^{21}$; nevertheless, it may be a reasonable explanation for these discrepancies.

Concern regarding the risk of worsening asystolic response by administering beta-blockers in patients with cardioinhibitory syncope ${ }^{22}$ was not supported, because the four patients with asystolic responses were well controlled by beta-blockers, without paradoxic responses.

In this study, a significant and impressive reduction in either syncopal or near-syncopal episodes was observed during long-term follow-up. It could be argued that the reduction in syncopal episodes was related to the patients' ability to abort the episode once diagnosis was confirmed instead of being a true pharmacologic effect. However, we believe that the concomitant reduction in near-syncopal episodes strongly suggests a true pharmacologic effect. Although placebo-controlled trials are required in patients with vasovagal syncope, the use of a placebo in patients with traumatic syncope is questionable. Treatment-related adverse events in our study were tolerable, and none of the patients required drug withdrawal.

\section{CONCLUSION}

In selected patients with malignant vasovagal syncope, treatment with metoprolol or propranolol at relatively high doses is feasible and, if guided by HUT results, is associated with a favorable outcome in terms of freedom from syncopal recurrences. 
1. Kenny RA, Ingram A, Bayliss J, et al. Head-up tilt: A useful tool for investigating unexplained syncope. Lancet. 1986;1:1352-1355.

2. Morillo CA, Klein GJ, Gersh BJ. Can serial tilt testing be used to evaluate therapy in neurally mediated syncope? Am J Cardiol. 1996;77:521-523.

3. Morillo CA, Leitch JW, Yee R, et al. A placebo controlled trial of intravenous and oral disopyramide for prevention of neurally mediated syncope. J Am Coll Cardiol. 1993;22: 1843-1848.

4. Moya A, Permanyer-Miralda G, Sagrista-Sauleda J, et al. Limitations of head-up tilt test for evaluating the efficacy of therapeutic interventions in vasovagal syncope: Results of a controlled study of etilefrine versus placebo. J Am Coll Cardiol. 1995;25:65-69.

5. Fitzpatrick AP, Ahmed R, Williams S, et al. A randomized trial of medical therapy for vasodepressor vasovagal syncope. Eur J Card Pacing Electrophysiol. 1991;2:43-48.

6. Brignole M, Menozzi C, Gianfranchi L, et al. A controlled trial of acute and long-term medical therapy in tilt-induced neurally mediated syncope. Am J Cardiol. 1992;70:339342.

7. Kapoor WN, Smith MA, Miller NL. Upright tilt testing in evaluating syncope: A comprehensive literature review. Am J Med. 1994;97:78-88.

8. Sutton R, Petersen M, Brignole M, et al. Proposed classification for tilt induced vasovagal syncope. Eur J Card Pacing Electrophysiol. 1992;2:109-113.

9. Fitzpatrick AP, Theodorakis G, Vardas $P$, et al. The incidence of malignant vasovagal syndrome in patients with recurrent syncope. Eur Heart $J$, 1991;12:389-394.

10. Decter BM, Goldner B, Cohen TJ. Vasovagal syncope as a cause of motor vehicle accidents. Am Heart J. 1994;127:1619-1621.

11. Sheldon R, Spalwinski J, Killman S. Reproducibility of isoproterenol tilt table test. Results in patients with syncope. Am $J$ Cardiol. 1992;69:1300-1305.

12. Grubb BP, Wolfe D, Temesy-Armos P, et al. Reproducibility of head-upright tilt table results in patients with syncope. PACE. 1992;15:1477-1481.

13. Chen XC, Chen MY, Remole S, et al. Reproducibility of head-up tilt table testing for eliciting susceptibility to neurally mediated syncope in patients without structural heart disease. Am J Cardiol. 1992;69:755-760.

14. Natale A, Akhtar M, Jazayeri M, et al. Provocation of hypotension during head-up tilt testing in subjects with no history of syncope or presyncope. Circulation. 1995;92:54-58.

15. Benditt DG, Petersen M, Lurie KG, et al. Cardiac pacing for prevention of recurrent vasovagal syncope. Ann Intern Med. 1995;122:204-209.

16. Sra JS, Jazayeri MR, Avitall B, et al. Comparison of cardiac pacing with drug therapy in the treatment of neurocardiogenic (vasovagal) syncope with bradycardia or asystole. NEJM. 1993;328:1085-1090.

17. Abboud FM. Neurocardiogenic syncope. NEJM. 1993;328:1117-1119.

18. Reu RF, Thames MD. Neural control mechanisms and vasovagal syncope. $J$ Cardiovasc Electrophysiol. 1993;4:587-595.

19. Lippman N, Stein KM, Lerman BB. Differential therapeutic responses of patients with 
isoproterenol-dependent and isoproterenol-independent vasodepressor syncope. $A m$ Heart J. 1994;128:1110-1116.

20. Grubb BP, Temesy-Armus $\mathrm{P}$, Hahn $\mathrm{H}$, et al. Utility of head upright tilt table testing in the evaluation and management of syncope of unknown origin. Am J Med. 1991;90:6-10.

21. Mahanonda N, Bhuripanyo K, Kangkagate C, et al. Randomized double-blind, placebocontrolled trial of oral atenolol in patients with unexplained syncope and positive upright tilt table test results. Am Heart J. 1995;130:1250-1253.

22. Dangovian HI, Jarandilla R, Frumin H. Prolonged asystole during head-up tilt table testing after beta-blockade. PACE. 1992;15:14-16. 